\title{
RESEARCH ON SPLICING METHOD OF DIGITAL RELIC FRAGMENT MODEL
}

\author{
Xuehui Yan $^{1}$, Yungang $\mathrm{Hu}^{1,2,3}, *$, Miaole Hou ${ }^{1,2,3}$ \\ ${ }^{1}$ School of Geomatics and Urban Spatial Informatics, Beijing University of Civil Engineering and Architecture, No.15Yongyuan \\ Road, Daxing District, Beijing, 102616-(yanxuehui , hyg, houmiaole)@ bucea.edu.cn \\ ${ }^{2}$ Beijing Key Laboratory For Architectural Heritage Fine Reconstruction \& Health Monitoring, No.15Yongyuan Road, Daxing \\ District, Beijing, 102616 \\ ${ }^{3}$ Engineering Research Center of Representative Building and Architectural Heritage Database, Ministry of Education, \\ No.15Yongyuan Road, Daxing District, Beijing, 102616
}

Commission III Urban Sensing and Mobility

KEY WORDS: Cultural Relic Fragments, 3D Model, Virtual Stitching

\begin{abstract}
:
In the course of archaeological excavation, a large number of pieces of cultural relics were unearthed, and the restoration of these fragments was done manually by traditional arts and crafts experts. In this process, cultural relics experts often try to splice the existing cultural relics, and then use adhesive to stick together the fragments of correct location, which will cause irreversible secondary damage to cultural relics. In order to minimize such damage, the surveyors combine 3D laser scanning with computer technology, and use the method of establishing digital cultural relics fragments model to make virtual splicing of cultural relics. The 3D software on the common market can basically achieve the model translation and rotation, using this two functions can be achieved manually splicing between models, mosaic records after the completion of the specific location of each piece of fragments, so as to effectively reduce the damage to the relics had tried splicing process.
\end{abstract}

\section{INTRODUCTION}

Cultural heritage is a symbol of national and national civilization and precious wealth. In the study of cultural heritage, many cultural relics of pottery and porcelain were broken up when they were unearthed. In order to study and restore complete cultural relics, many archaeologists use traditional process ontology of cultural relics in hand stitching. Although this can be directly recovered cultural relics, but in the process of restoration, the irreversible secondary damage to the cultural relics comes from the fact that in order to determine the correct spatial location of the cultural relics, as well as the trial splicing and adhesives. This does not conform to the principle of protection centered on the safety of cultural relics. With the progress of the times and the rapid development of science and technology, this phenomenon has been improved by the appearance of non-contact measurement. Among them, the most widely used one is a three-dimensional laser scanner. Combined with the later computer data processing software, a refined digital 3D model of cultural relic fragments can be obtained. If these digital models can be used in the computer to process virtual stitching with the model processing software, it can avoid damage to cultural relic fragments effectively.

In Emperor Qinshihuang's Mausoleum Site Museum, the archaeologists every time discovering a large number of incomplete terracotta pieces, there are few terracotta bodies are unearthed. For these fragments, archeologists need to manually splicing and restoring, but this method requires a lot of manpower and material resources, a long time, low efficiency, and easy to cause secondary damage to the precious cultural relic fragments. In particular, due to the local natural environment (temperature, humidity), only five months each year are suitable for the splicing of the Terracotta Army. Moreover, whether the mechanical reinforcement or adhesive reinforcement used in the splicing process will cause irreversible damage to the Terracotta $\operatorname{Army}(\operatorname{Lan}, 2001)$, it is necessary to ensure that the shards are properly spliced in one shot. It has been nearly 40 years since the excavation of Emperor Qinshihuang's Mausoleum Site Museum. However, the fully recovered Terracotta Army only accounted for a small part. Most of them are still waiting to be restored. How to rehabilitate the cultural relic fragments safely and efficiently, make them exhibit the characteristics of the original features, and spread their historical values is a problem that needs to be solved urgently, and has far-reaching research value and significance.

\subsection{Research Status}

There are many research results for the splicing of the digital cultural relic fragments model, and different algorithms have been developed. According to some characteristics of cultural relic fragments, such as the shape, the thickness, texture or coloured drawing on the surface, there are corresponding stitching algorithms. The basis of splicing is mainly the extraction of feature information. The feature information includes contour lines, fracture surfaces, and surface texture. Use outer and inner edges to extract the outer contour, using normal angle calculation and statistical methods to determine the fracture surface, Using the scanning line algorithm to obtain the inner contour line, proposes the feature contour line extraction method of triangular mesh surface model(Fan, 2005). The feature contours of the crushing rigid body model were successfully extracted to enable spatial curve matching.

\footnotetext{
* Corresponding author: hyg@ bucea.edu.cn (Yungang Hu)
} 
1.1.1 Matching Curves based on Model Contours: The contour lines of the model include the edge contour of the model and the contour line of the model section. Profile-based curve matching can be roughly divided into two categories from the application point of view. First, for thin-walled cultural relic fragments such as pottery and murals, the outline of the cultural relic fragments model is used as a feature to match the debris to a two-dimensional plane curve. The second is to match the three-dimensional space curves of the fracture surface contours of non-thin-wall cultural relic fragments based on the matching of two-dimensional plane curves.

Analyze the three-dimensional object's fragments contour curve, study multi-scale wavelet contour descriptors, and propose a multi-scale contour curve feature extraction and threedimensional object fragmentation method based on multi-scale analysis(Zhou, 2006).

1.1.2 Surface Matching based on Fracture Surface: A piece of cultural relic fragments that can not be ignored in thickness. Using the edge of the 3D model as the boundary, the surface of the model is first segmented into multiple curved surface, and the fracture surface is identified from it. Then, according to the feature of the extracted fractured curved surface, the matching is performed, so as to get the $3 \mathrm{D}$ rigid transformation matrix of the debris matching, so that the common parts of the two surfaces located in different coordinate systems can be correctly matched.

The algorithms for matching surfaces based on local features include geometric hashing(Pan, 2004. Ran, 2006), clustering algorithm(Xiaoa, 2005), genetic algorithm(Wang, 2007), and random sampling consistency algorithm(Winkelbach, 2008).

Qi Shixia proposed a fault surface matching algorithm based on feature region matching. First, the concave and convexity of each vertex of the fracture surface is calculated. According to the result, the surface vertices are classified and connected to generate the characteristic region. Then PCA principal component analysis method is used to calculate similar feature regions, and the similarity feature regions are subjected to compatibility constraints and error metrics, so that the optimal feature region matching pair set achieves initial matching alignment(Qi, 2015).

The most widely used and most classical surface matching technique is the iterative closest point matching (ICP) algorithm This algorithm has a monotonic convergence to the model, but it is easy to get a local optimal solution using this algorithm(Besl, 1992). Based on this algorithm, later generations have developed some improved ICP algorithms. These matching techniques are based on the model's overall optimal fusion criteria to perform data iterations one after another, so as to optimize the matching results.

In general, different algorithms have different advantages and disadvantages. At present, complete and partial matching of complex fragments can be achieved, but the results may also lead to incorrect matching $(\mathrm{Li}, 2012)$.
1.1.3 Matching based on Surface Texture Information: Among such algorithms, the most classic is the color-based ICP (Color ICP) algorithm proposed by Johnson. It combines the three-dimensional geometric information of the object with the color, which greatly improves the registration fusion efficiency and accuracy of the three-dimensional model.

Northwest University took the lead in domestic research on the recovery of crushing rigid bodies and proposed a variety of model processing algorithms. Fan Shaorong proposed the concept of the inner and outer contours of the fragments 3-D model, and presented the complementary shape matching method under the constraint of the cross-boundary tangent vector. Yuan Shaofeng proposed a model approximation method of polygon approximation, and based on this, studied two-dimensional and three-dimensional multi-angle arc matching algorithms respectively. Zhou Shucheng described the contour of the model with wavelet transform for thin-walled artifacts, and used this descriptor to match curves. Aiming at the fragmented model with thickness, a matching method based on fracture surface is proposed, and the matching of cultural relic fragments model is realized by direction mapping. Li Qunhui segmented the model fracture surface based on the integral invariant, and used a search method based on similar constraints of model triangle patches to find the optimal matching of the fracture surface.

Pan Xiaolin of Nanjing University of Aeronautics and Astronautics has developed a general model surface matching algorithm and used a double-level hash voting mechanism to calculate the results of model matching. Based on the directed depth image, Wang Jian transforms the matching problem of the 3D model into the comparison of the 2D spatial image, and uses the genetic algorithm to find the optimal position for the surface matching in the 7-dimensional space. Zhu Yanjuan proposed a registration algorithm based on point cloud model. Calculate the normal point of the surface of the feature point and the curvature to find matching pairs. Then use the geometric hashing method for initial registration, iterative closest point algorithm (ICP) is used to perform secondary registration of the model. Effectively solve the ICP algorithm on the model of the initial position of the problem.

Li Meng of Harbin Institute of Technology proposed two kinds of feature descriptors based on vector representation and spherical sigma in the extraction of three-dimensional models. They describe the feature points and feature shapes of the threedimensional model respectively. Proposed an area segmentation algorithm based on the height map of fragmented fracture surface in the aspect of three-dimensional model surface segmentation. Based on the regional segmentation, the height map of the fracture surface of the debris model is used as the matching feature, and the degree of complementarity between them is used as the matching criterion to realize the splicing restoration of the debris.

Pan Rongjiang from Shandong University did a detailed study on how to find local matching relationships. A threedimensional model boundary extraction algorithm based on the maximum circle is given, and a method of splicing rotating type debris is proposed. It transforms the splicing relationship between two fragments into a geometric optimization problem concerning the two parameters of translation and rotation.

In the current research, there are many research on automatic stitching algorithm. It can splice the adjacent pieces by 
algorithm, but it will produce more errors with each iteration, and gradually affect the accuracy of stitching. In addition, the research also only mentioned on the algorithm, and no algorithm is applied in practical project, there is no one algorithm developed automatic stitching software can be extended based on that in the model fragment reassembly field, no one can really apply and solve the practical problems of software.

\section{CULTURAL RELIC FRAGMENTS MODEL SPLICING TECHNOLOGY ROUTE}

\subsection{Splicing data preparation and requirements}

For cultural relic fragments, the process of modeling using 3D laser scanning technology is an inverse modeling process. Reverse engineering software functions are usually focused on processing and optimizing dense scan point clouds to generate more regular result point clouds. The regular point cloud can be applied to rapid prototyping, or the final NURBS surface can be constructed from these regular point clouds to be imported into CAD software for subsequent structural and functional design work.
Taking the Terracotta Armys as an example, after obtaining the cloud point data of the field scan, the triangulation model of the fragments needs to be constructed through reverse modeling software. The software directly generates models with many problems, such as noise and holes. And so on, the data needs to be preprocessed manually before it can be applied to the next stitching process.

2.1.1 Data Collection: To build a digital model of cultural relic fragments with color texture like Terracotta Army fragments, two aspects should be taken into account when collecting data, that is, the geometric information of the fragments and the texture color information of the fragments. When collecting the geometric three-dimensional digitized information of the Terra Cotta Warriors, the Hexagon articulated arm is used. The instrument has high precision and dense point clouds, and is suitable for collecting digitized information of cultural relic fragments with high precision. At the same time, texture information was collected using a Nikon D4 full-frame SLR camera.

2.1.2 Data Processing: The collected point cloud data also needs to be pre-processed by the data to perform spatial 3D modeling. The specific modeling process is shown in Figure 1.

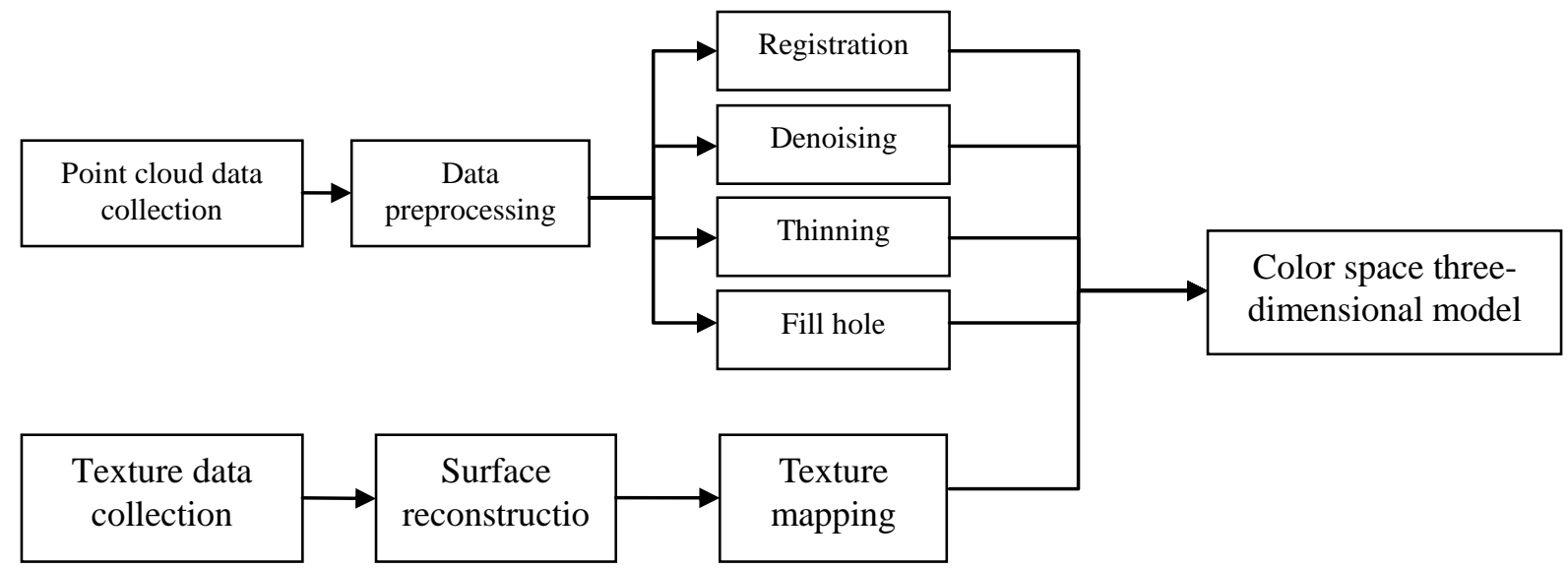

Figure 1 Cultural fragments digital flow chart

For the needs of cultural relics display, the fragmented digital model will first be stitched in a virtual way on the computer. In this way, not only can the cultural relics worker provide a spatial reference when repairing, the number of trial splicing operations can be greatly reduced, and secondary damage to cultural relic fragments during the repair process can be effectively reduced.It can also be used to create a complete picture of a man, to provide convenience for later exhibitions.

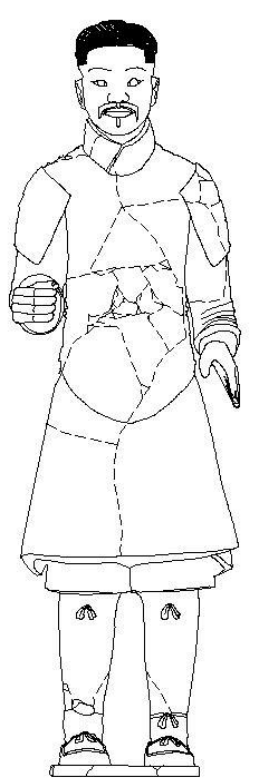

Figure 2 Line Map of Terracotta Army 


\subsection{Splicing Principle}

Since the manual splicing is used, the splicing principle is simpler than the automatic splicing. However, the advantage of manual splicing is that it can control the precision very well, and it will not cause the shards to produce larger errors after multiple iterations of splicing.

In archaeological and cultural restoration, professionals need to use the six views of the relics, namely, front view, rear view, left view, right view, top view and bottom view. This requires the relics to be placed in the coordinate system in the frontal view. When using the scanner to collect data, it is impossible to control the spatial position and orientation of the threedimensional data, which requires us to rotate, translate, and scale the geometric coordinates of the data so that they exist in the coordinate system with the correct orientation. Usually based on the head, the sorted fragments are spliced together one by one to obtain a complete human splicing model.

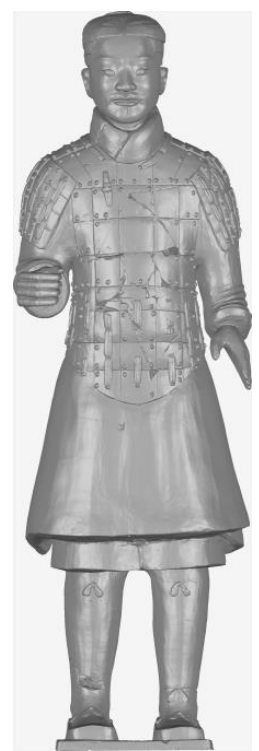

Figure 3 Front view of Terracotta Army

\subsection{Stitching Process}

After the three-dimensional digitized model of the Terracotta Army and pelicans fragment was established, all the fragments of an entire human skeleton were numbered first, and the corresponding attributes of each fragment were recorded, such as the size of the fragment, the surface texture features, and the color painting and other information. After that, according to these attribute information, the classification of the Terracotta Army to which the fragments belong can be roughly divided into five parts, namely, head and neck, armorpieces, war dresses, limbs, and other indiscernible small pieces. After that, the classification result of the fragment is also associated as an attribute information to the corresponding fragment.

After all preparations are completed, the fragment data can be imported into the self-developed software to start splicing. First splicing parts according to the classification results, eliminating unnecessary processes that are repeatedly selected during direct splicing. Get a relatively complete chunk model for each part, and fix the relative positions of each fragment inside each chunk model. Afterwards, several large-scale models were integrated and integrated into one person. Finally, in the process of classification in the previous step, it is possible that the remaining small pieces, which are difficult to distinguish the specific location, are embedded in the crevices of the human body, and a complete human skeleton can be obtained.

Unlike automatic stitching, manual stitching requires more manual intervention. The connection between debris and debris is all determined by visual observation. In order to reduce artificial labor and speed up splicing, the debris model can be classified first, then spliced according to different categories, and finally integrated. The complete cultural artifact model thus obtained can not only ensure the accuracy of splicing, but also guarantee the splicing speed. 


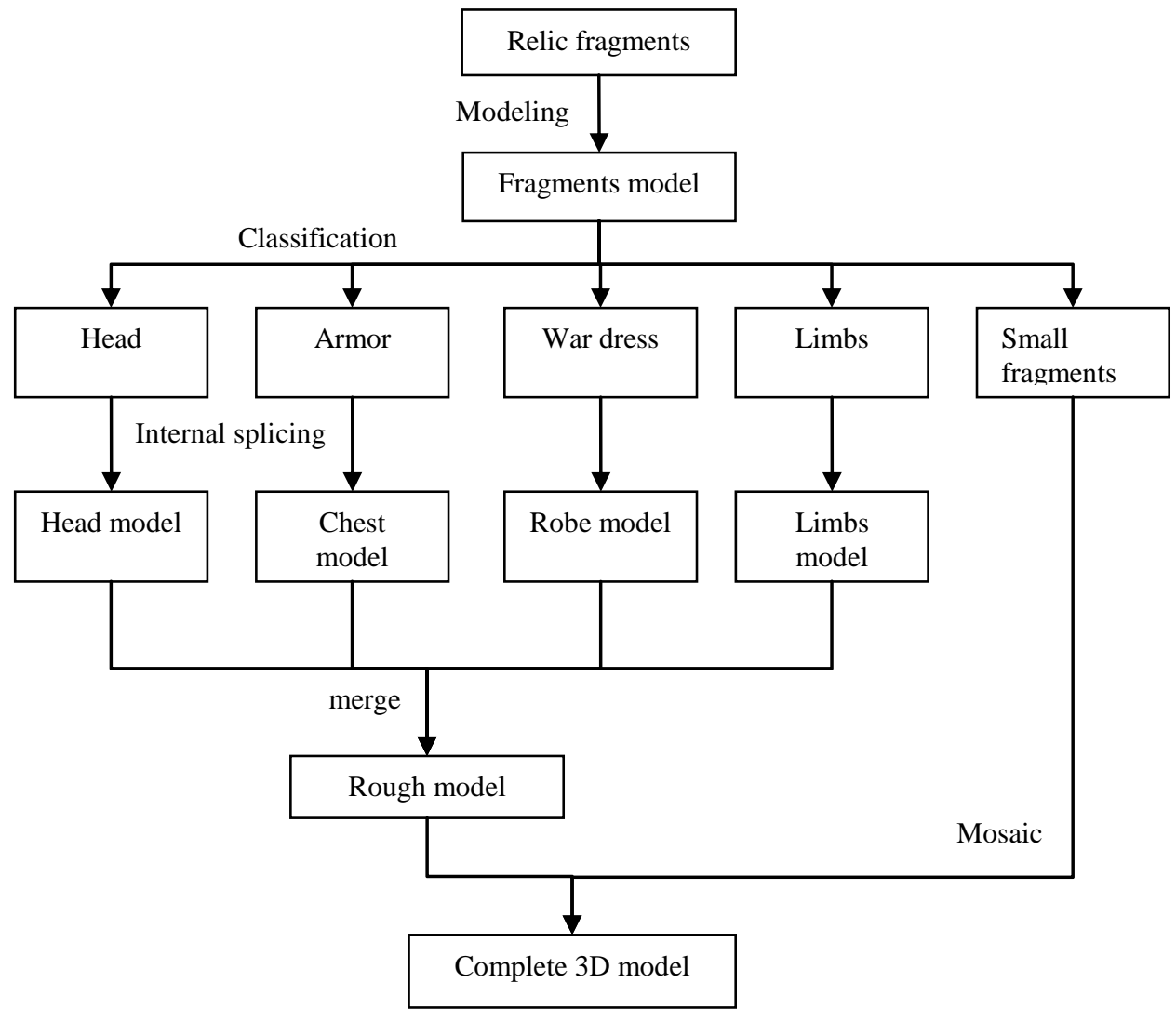

\subsection{Classification Number of Fragments}

Taking the splicing model of Terracotta Army as an example, classification can be done according to the characteristics of different parts of the model. The head is equipped with ropes, cards, hairpins and other features. The chest and abdomen are easy to identify with armor and armor. The lower body has a smooth surface with rounded edges. The extremities have cylindrical shapes with similar diameters. The hands and feet also have obvious features and can be easily identified.

According to these characteristics, each person's skeleton fragments are classified. Combining the human body structure and the actual shape of the Terracotta Army, the fragments can be divided into four categories: head, armor, war dress, and limbs, as well as some small fragments that are too fine to be distinguished.

When the Terracotta Army fragments are unearthed, they will be numbered in accordance with the position (pit number, channel number, sequence number) and the order of unearthing of the person's skeleton. However, this number is not conducive to the classification of fragments splicing. On the basis of this numbering, we replace the unearthed portion of the original number with the category to which the fragment belongs and the natural number in the classification, to obtain such a number: pit number - channel number - sequence number - fragment type - number. At the same time, in order to facilitate future cultural relics' splicing of cultural relics according to model mosaic results, the original fragment serial number will be connected as a piece of attribute information to corresponding fragments, which can be viewed by cultural relics workers when they are needed.

\subsection{Fragment Location Description}

After acquiring the data of the Terracotta Army using a threedimensional laser scanner, the data of each fragment can be defined as a spatial entity. The expression of the spatial entity is spatial data, and the spatial data is used as the basis for the splice work.

The point cloud data acquired by the three-dimensional laser scanner all have coordinate information based on the coordinates of the instrument itself. Therefore, the model of the Terracotta Army is actually in a unified coordinate system. As long as coordinate adjustment is performed for each piece of debris on the basis of this coordinate, the position of the model can be changed to achieve the splicing effect. The scanned data has real size information, so the adjustment of the coordinates is limited to rotation and translation, which can be expressed using a complex matrix(Shen, 2011):

$$
\begin{gathered}
{\left[\begin{array}{l}
u \\
v \\
w \\
l
\end{array}\right]=\left[\begin{array}{llll}
1 & 0 & 0 & x_{0} \\
0 & 1 & 0 & y_{0} \\
0 & 0 & 1 & z_{0} \\
0 & 0 & 0 & 1
\end{array}\right]\left[\begin{array}{ccccc}
1 & 0 & 0 & 0 \\
0 & \cos \alpha & -\sin \alpha & 0 \\
0 & \sin \alpha & \cos \alpha & 0 \\
0 & 0 & 0 & 1
\end{array}\right]\left[\begin{array}{cccc}
\cos \beta & 0 & \sin \beta & 0 \\
0 & 1 & 0 & 0 \\
-\sin \beta & 0 & \cos \beta & 0 \\
0 & 0 & 0 & 1
\end{array}\right]\left[\begin{array}{cccc}
\cos \gamma & -\sin \gamma & 0 & 0 \\
\sin \gamma & \cos \gamma & 0 & 0 \\
0 & 0 & 1 & 0 \\
0 & 0 & 0 & 1
\end{array}\right]\left[\begin{array}{l}
x \\
y \\
z \\
l
\end{array}\right]} \\
\text { Figure 5 Complex matrix }
\end{gathered}
$$




\section{PROGRAMMING}

In response to the need for debris splicing at the Emperor Qinshihuang's Mausoleum Site Museum and the fact that humans directly caused damage to the cultural relics by splicing directly on the physical basis, we developed a splicing software based on MeshLab. The manual splicing of the Terracotta Army models can be done manually, and each shard after splicing has its own attribute data, which can record the correct relative spatial position and the number of adjacent shards. This allows artifact restoration workers to directly perform physical restoration of cultural relic fragments based on the data provided by us. It eliminates the secondary damage caused by

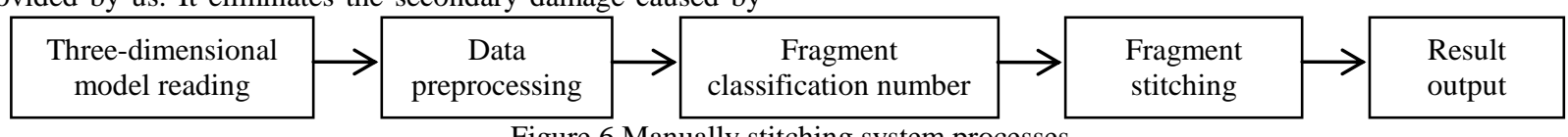

Figure 6 Manually stitching system processes

The system adopts a modular design concept and divides the system into three major modules:

(1) Three-dimensional model module responsible for reading and displaying the model.

(2) The fragment splicing module, which is further subdivided

nto three small modules, namely, a data pre-processing module, a fragment classification module, and a fragment splicing module.

(3) The results display module mainly completes the display of the splicing results, saves the output, and can be saved as a common obj format. the cultural relics during the trial splicing process and improves efficiency.

\subsection{System Flow and Module Display}

When designing the system, full consideration was given to system availability. Under the premise of ensuring the function, the system operation is simplified as much as possible and the operation difficulty is reduced. Based on this goal and combined with archaeological needs, the final system flow is as follows:

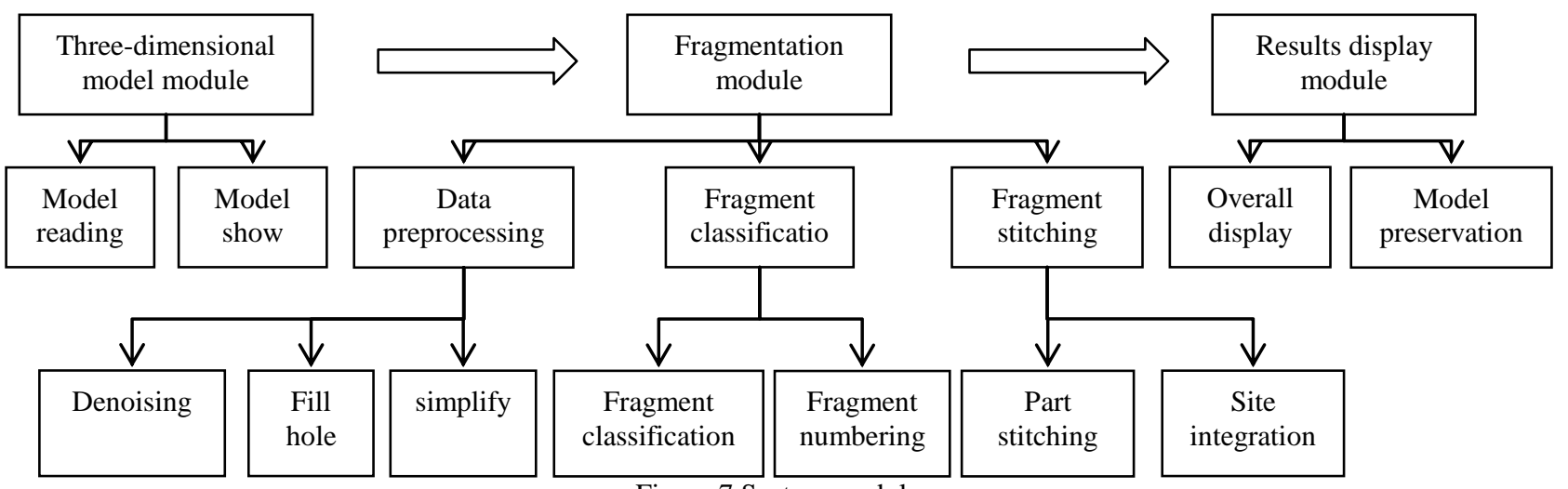

Figure 7 System module

\subsection{System Display}

multiple models can be displayed at the same time. The splicing can be performed using the rotation and translation tools.

The part of the 3D model module is set to read only the obj format model, avoiding errors that may occur during format conversion. After the model is opened, the model can be rotated, translated, etc. in the current interface.

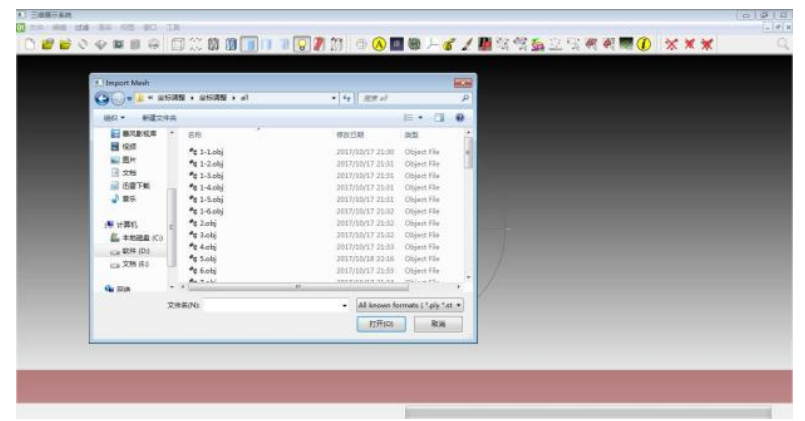

Figure 8 Open a file

The fragmentation module has three categories of preprocessing classification and splicing. The preprocessing menu includes three functions: denoising, hole filling, and simplification. The fragments can be divided into one of the five categories and numbered. After entering the splicing section,

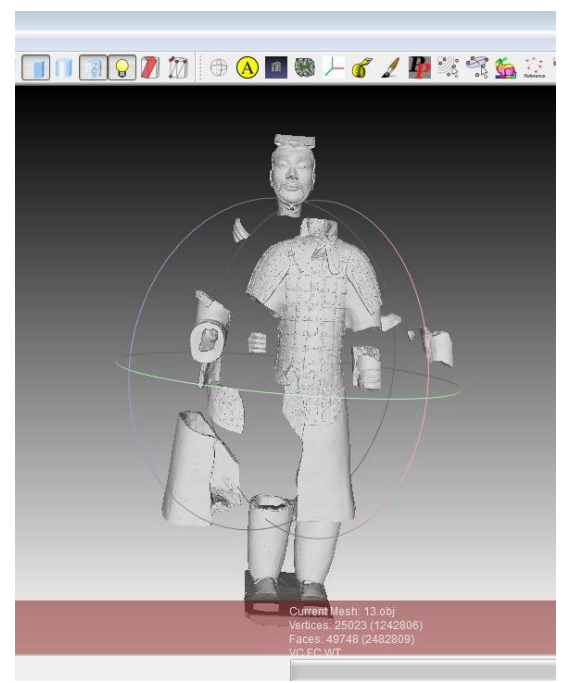

Figure 9 Fragment stitching 


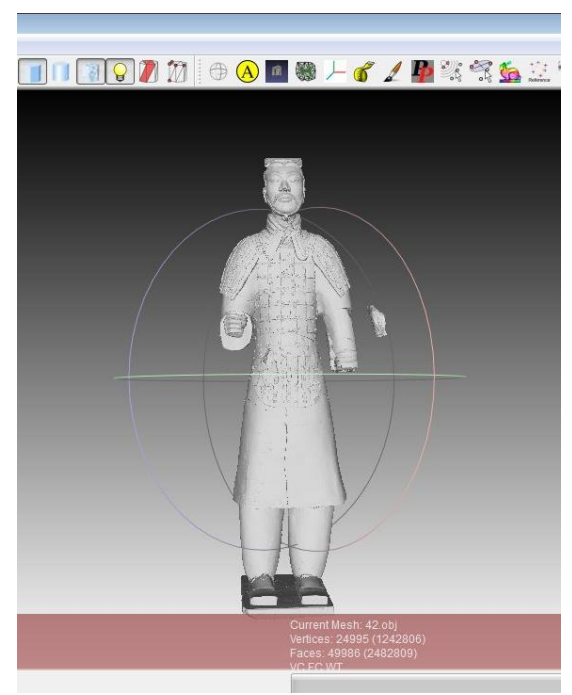

Figure 10 Splicing products

After the stitching is completed, the new coordinate information of the model is saved to ensure the correct relative position when opened in other software.

\section{SUMMARY AND OUTLOOK}

For the splicing of fragments, using the existing threedimensional software, whether it is a forward modeling software or an inverse modeling software, manual splicing of the model can be achieved.However, what the existing modeling software on the market can not achieve is to attach attributes to the model. With these additional attributes, the spatial position information after fragmentation can be fully recorded. The accuracy of this splicing method completely depends on the judgment of the human eye, it is precisely because of this that the spliced model does not appear to be visible to the naked eye when it is used and displayed, thus achieving a visually perfect effect. Manual splicing takes time and effort. In particular, it requires the operator to align each gap with patience and meticulously, otherwise a slight deviation will lead to huge gaps between the two pieces in the later period.

Although this method of manual splicing using software is effective, but the era is developing, technology is advancing, or it should actively develop more intelligent automatic splicing software to complete this work.

\section{REFERENCES}

Besl, P. J., \& Mckay, N. D. (1992). A Method for Registration of 3-D Shapes. IEEE Computer Society.

Fan, S. (2005). A method of extracting feature contour from triangular mesh surface model of fractured solid. Journal of Computer Aided Design \& Computer Graphics, 17(9), 20032009.

Lan, D. (2001). Talking about the Repair of Qin Terracotta Army. wenbo (5), 72-75.

Li, Q., Zhou, M., \& Geng, G. (2012). Fractured Surfaces Matching for Reassembling Broken Solids. Fifth International Symposium on Computational Intelligence and Design (Vol.2, pp.511-514). IEEE Computer Society.
Pan, X. L., Zhang, L. Y., Jie, Y. W., \& Zhu, Y. J. (2004). Algorithm for three-dimensional partial surface matching. Journal of Nanjing University of Aeronautics \& Astronautics, 36(5), 544-549.

Qi, S. (2015). Study on Patching Method of Gentiana Macrophylla Based on Fracture Surface Matching. (Doctoral dissertation, Northwest University).

Ran, G., \& Cohen-Or, D. (2006). Salient geometric features for partial shape matching and similarity. Acm Transactions on Graphics, 25(1), 130-150.

Shen, Y. (2011) Research and Implementation of Automatic Archeological Drawing Method Based on 3D Model of Cultural Relics. (Doctoral dissertation, Northwest University).

Wang, J., Zhou, L. S., Zhang, L. Y., \& Zhu, Y. J. (2007). Surface matching based on genetic algorithm. Journal of Image \& Graphics, 12(4), 695-699.

Winkelbach, S., \& Wahl, F. M. (2008). Pairwise matching of 3d fragments using cluster trees. International Journal of Computer Vision,78(1), 1-13.

Xiaoa, G., Onga, S. H., \& Foongc, K. W. C. (2005). Efficient partial-surface registration for $3 \mathrm{~d}$ objects. Computer Vision \& Image Understanding, 98(2), 271-293.

Zhou, S., Geng, G., \& Zhou, M. (2006). Multiscale method for mosaicing 3d fragmented objects. Journal of Computer-Aided Design \& Computer Graphics. 